\title{
LOW LEVEL LASER THERAPY VERSUS DRY NEEDLING FOR INACTIVATION OF MYOFASCIAL TRIGGER POINTS
}

\author{
Sherif Mohamed Ezzat Sayed", Hamida Refai* ${ }^{* *}$, Nermine Hassanin ${ }^{* * *}$ and Ahmed Abbas Zaky ${ }^{* * * *}$
}

\begin{abstract}
The aim of the present study was to evaluate and compare two forms of treatment for inactivation of MTrPs: laser therapy and dry needling. This study included 17 females and 1 male patients with active myofascial trigger points of masseter muscle of ages ranged from 18 to 42 years with an average of 30 years divided into 2 equal groups, in the $1^{\text {st }}$ group the MTrPs received application of low level laser (diode laser of wavelength 980 nanometers, power 0.2 watt, total energy 12 joule and exposure time 50 seconds) while in the $2^{\text {nd }}$ group the MTrPs underwent dry needling. Both groups underwent 3 treatment sessions a week for 4 weeks; each session took 50 seconds and were assessed for the pain intensity at rest, on function and on palpation [PP] measured by NRS and the painless MMO. All were measured prior to the treatment, at the end of 2- weeks (mid-treatment), at the end of 4- weeks (end of treatment), and monthly after completion of treatment for 3 months. The results of the present study demonstrated that all evaluated parameters showed insignificant differences between both groups throughout the study intervals. However, laser group showed statistically significantly higher mean $\%$ decrease in pain scores at rest at the end of treatment than dry needling group. While at the end of the study, dry needling group showed statistically significantly higher mean \% decrease in pain scores on function than Laser group. At the end of treatment, laser group showed statistically significant analgesic effect of LLLT. With the exception of statistically significant decrease in mean PP scores, dry needling group showed insignificant decrease in pain intensity at the end of treatment. There was no statistically significant change in mean MMO through all periods in both groups. By the end of the study, all the evaluated parameters showed insignificant difference relative to the baseline with the exception of the significant decrease in mean PP scores in dry needling group.
\end{abstract}

KEYWORDS: Myofascial Trigger Points, Low Level Laser, Dry Needling.

* B.D.S. 2005, Faculty of Oral and Dental Medicine, Cairo University.

** Professor of Oral and Maxillofacial Surgery. Faculty of Oral and Dental Medicine, Cairo University. *** Ass. Prof. of Oral and Maxillofacial Surgery. Faculty of Oral and Dental Medicine, Cairo University. **** Ass. Prof of Dental Laser Application, National Institute of Laser Enhanced Sciences (NILES), Cairo University. 


\section{INTRODUCTION}

Myofascial pain syndrome (MPS) is one of the most frequent causes of pain involving the orofacial region. ${ }^{(1)}$ It arises from muscles or related fascia and is usually associated with myofascial trigger points (MTrP). Certain diagnostic criteria have been reported, such as a palpable and hypersensitive taut muscle band, if the muscle is accessible, recognition of pain by the patient and soreness when the affected muscle is stretched. (2) Therefore, diagnosis is purely clinical, based on a detailed history and thorough physical examination performed by muscle palpation and observation of motor function. ${ }^{(3)}$

Trigger points are classified as being active or latent, depending on their clinical characteristics. ${ }^{(4)}$ When these points are active, patients often complain of pain distant from the site, e.g., in the head, ear, mandible, temporomandibular joint (TMJ), teeth, eyes and cervical spine. Active trigger point causes pain at rest and it is tender to palpation with a referred pain pattern that is similar to the patient's pain complaint. ${ }^{(4,5,6)}$ This referred pain is felt not at the site of the trigger-point origin, but remote from it. The pain is often described as spreading or radiating. ${ }^{(7)}$ Referred pain is an important characteristic of a trigger point and it differentiates a trigger point from a tender point, which is associated with pain at the site of palpation only. ${ }^{(8)}$

No consensus has yet been reached regarding the etiology of MPS. macrotrauma or repeated microtrauma caused by parafunctional, structural disharmonies, such as occlusal disorder, continuous source of deep pain and emotional distress were implicated to play a role its pathogenesis. Given its multifactorial etiology, no standard treatment protocol for MPS is currently available. (3) Instead, several treatment alternatives have been suggested.

Numerous treatment modalities have been evolved for deactivation of MTrPs include ultrasound $^{(9)}$, application of pressure or massage ${ }^{(10)}$, transcutaneous electrical nerve stimulation $(\text { TENS })^{(11)}$, ethyl chloride spray and stretch techniques $^{(12)}$, acupuncture ${ }^{(3)}$, dry needling ${ }^{(13-15)}$, injection of certain agents such as local anesthetic and botox $^{(16)}$, and low-level laser therapy ${ }^{(17-19)}$. It is of paramount importance to clinically assess and compare some of the therapies used for MTrPs deactivation with a view for establishing more effective alternatives for the treatment of MTrP. The aim of the present study was to evaluate and compare two forms of treatment for inactivation of MTrPs: laser therapy and dry needling.

\section{PATIENTS AND METHODS}

Eighteen patients with active myofascial trigger points of masseter muscle who meet the inclusion criteria participated in this study. They were 17 females and 1 male. Their ages ranged from 18 to 42 years with an average of 30 years. After accepting to be enrolled in the present study, each patient was assigned into one of 2 equal-sized groups, Group 1: The trigger points in nine patients received application of low level laser (diode laser of a wavelength 980 nanometers, power 0.2 watt, total energy 12 joule and exposure time 50 seconds). Group 2: Nine patients underwent dry needling of MTrPs. MTrPs in both groups underwent 3 treatment sessions a week for 4 each session took 50 seconds.

\section{Identification of Trigger points and Assesement of mouth opening}

Each patient rated the pain and the tenderness (pain on palpation $[\mathrm{PP}]$ ) on numerical rating scale (NRS) of 0-10 with zero being no pain and ten correspond to the worst pain that the patient ever had. Assessment of painless maximum mouth opening (MMO) was performed by measuring the distance in $\mathrm{mm}$ between the incisal edges of the upper and lower central incisors using Vernier graduated caliper. 


\section{Low Level Laser (diode) application}

After localization of the MTrP, the taut muscle band was marked. The diode laser- probe was applied over the $\operatorname{TrP}$ with "scanning" movements. (Fig. 1).

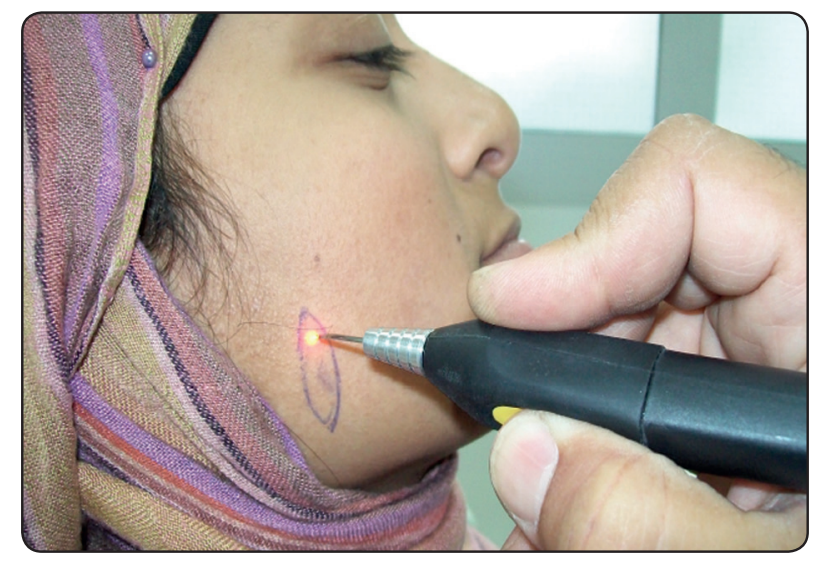

Fig. (1): Application of laser to the MTrP

\section{Technique of Dry needling}

The taut band was marked, cleansed with a Betadin* swab and localized between the thumb and the index finger. It was needled forward and backward repeatedly using hypodermic needles of $23 \mathrm{~g}$, x1.5 inch needle with $3 \mathrm{ml}$ disposable plastic syringe. The needle was inserted to a depth of 1- 2 $\mathrm{cm}$ at an acute angle of $30^{\circ}$ to the skin, in various directions, with movement into the tissue.

\section{Postoperative care and instructions}

The patients were advised to do physiotherapy triple a day (open and close his mouth 10 times at morning, afternoon and night) 7 days a week. Warm fomentation 3 or 4 times a day was applied on the affected area.

\section{Postoperative Evaluation}

Outcome measures included assessment of the pain intensity at rest, on function and on palpation
[PP] of the MTrP measured by NRS as well as assessment of the painless MMO. These outcome variables were measured prior to the treatment, at the end of 2-weeks (mid-treatment), at the end of 4- weeks (end of treatment), and monthly after completion of treatment for 3 months. The collected data was then statistically analyzed.

\section{RESULTS}

The results of the present study demonstrated that all evaluated parameters showed insignificant differences between both groups throughout the study intervals. However, laser group showed statistically significantly higher mean $\%$ decrease in pain scores at rest at the end of treatment than dry needling group. While at the end of the study, dry needling group showed statistically significantly higher mean $\%$ decrease in pain scores on function than Laser group. At the end of treatment, laser group showed statistically significant analgesic effect of LLLT. With the exception of statistically significant decrease in mean PP scores, dry needling group showed insignificant decrease in pain intensity at the end of treatment. There was no statistically significant change in mean MMO through all periods in both groups. By the end of the study, all the evaluated parameters showed insignificant difference relative to the baseline with the exception of the significant decrease in mean PP scores in dry needling group.

\section{Subjective findings}

Pain at rest There was no statistically significant difference between $\%$ decrease in pain scores of the two groups through all periods except after 4 weeks where Group I showed statistically significantly higher mean $\%$ decrease in pain scores than Group II.

\footnotetext{
" Betadin: povidone-iodine USP Nile Pharmaceuticals Co., Cairo, Egypt.
} 
Pain during function After 2 weeks and after 4 weeks; Group II showed statistically significantly higher mean \% decrease in pain scores than Group I while after 1 month, Group I showed statistically significantly higher mean $\%$ decrease in pain scores than Group II. After 2 months, there was no statistically significant difference between the two groups while after 3 months; Group II showed statistically significantly higher mean $\%$ decrease in pain scores than Group I (Fig. 2).

\section{Objective Findings}

Pain on palpation [PP] In Group I, there was non-statistically significant decrease in mean pain scores after 2 weeks and after 3 months but there was a statistically significant decrease in mean pain scores through all other periods. In Group II, there was non-statistically significant decrease in mean pain scores after 2 weeks while there was a statistically significant decrease in mean pain scores through all other periods (Tab 1).

Maximum painless mouth opening There was no statistically significant difference between the two groups through all periods.

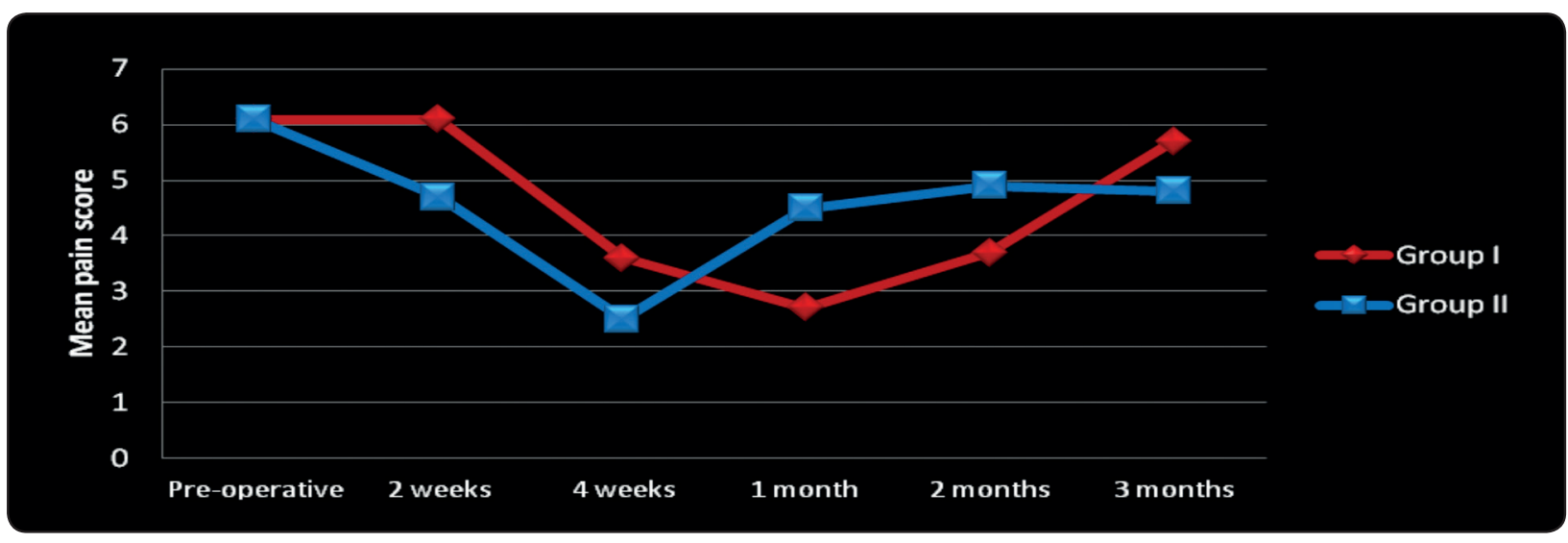

Fig. (2): Line chart representing changes by time in pain scores during function in each group.

TABLE (1) The mean differences, standard deviation (SD) values and results of Wilcoxon signed-rank test for the changes by time in mean PP scores in each group

\begin{tabular}{|c|c|c|c|c|}
\hline Group & Period & Mean difference & SD & P-value \\
\hline \multirow{4}{*}{ I } & Pre-operative -2 weeks & -1.6 & 2.6 & 0.055 \\
\cline { 2 - 5 } & Pre-operative - 4 weeks & -3.6 & 3.2 & $0.004^{*}$ \\
\cline { 2 - 5 } & Pre-operative - 1 month & -3.4 & 3.3 & $0.006^{*}$ \\
\cline { 2 - 5 } & Pre-operative - 2 months & -1.9 & 2.7 & $0.028^{*}$ \\
\cline { 2 - 5 } & Pre-operative - 3 months & -1.1 & 2.2 & 0.095 \\
\hline \multirow{5}{*}{ II } & Pre-operative - 2 weeks & -0.7 & 1.8 & 0.131 \\
\cline { 2 - 5 } & Pre-operative - 4 weeks & -3.1 & 3 & $0.004^{*}$ \\
\cline { 2 - 5 } & Pre-operative - 1 month & -2.9 & 2.7 & $0.006^{*}$ \\
\cline { 2 - 5 } & Pre-operative - 2 months & -2.4 & 2.6 & $0.007^{*}$ \\
\cline { 2 - 5 } & Pre-operative - 3 months & -2.4 & 2.5 & $0.008^{*}$ \\
\hline
\end{tabular}




\section{DISCUSSION}

The myofascial trigger point (MTrP) is the hallmark physical finding of the myofascial pain syndrome (MPS) which is a common source of discomfort and disability for many patients; however, it is generally ignored or misdiagnosed leading to chronic painful conditions. There is no standard therapeutic protocol for the treatment of MPS and different alternatives have been suggested. The main goal to be achieved is restoration of complete range of motion, along with the identification and removal of perpetuating factors and inactivation of MTrPs. The aim of the present study was to evaluate and compare two forms of treatment for myofascial pain; laser therapy and dry needling since there is a lack of clinical trials comparing the two techniques.

Since MTrPs offer few objective clinical variables that lend themselves to sequential followup, in order to evaluate the patients' response to treatment we chose a number of validated subjective outcome measures that reflect pain, tenderness and limitation of mouth opening. The choice of the patients and the fixed parametrers were important to reach a standard result that's why, in the present study, the patients were chosen with no significance difference in age, gender and MTrPs' number and site in both groups.

The primary outcome of interest was the change in pain from baseline to endpoint. In the current study, Laser group showed significant decrease of pain scores at rest, on function and on palpation (PP) of the MTrP at the end of treatment. Various explanations for the analgesic effect of LLLT have been suggested. According to Simunovic ${ }^{(20)}$, LLLT improves local microcirculation, favors the supply of oxygen to cells with hypoxia and helps remove the waste products of cell metabolism, thereby breaking the vicious cycle of pain, muscle spasm and further pain. Ozdemir et al ${ }^{(21)}$ suggested laser irradiation provides analgesia by decreasing the spasm in muscle arterioles which is essential for tissue oxygenation and by increasing ATP formation with a consequent normalization in metabolic rate of the tissues with diminished energy levels. The other mechanisms may be related with its effects on endorphin levels and gate control of pain. By all these mechanisms it can interrupt the vicious cycle of the trigger point. ${ }^{(22)}$

In the present study, the intensity of pain was assessed at rest, on function and on palpation (PP) of the MTrP. The statistically significant analgesic effect of LLLT at the end of treatment is in accordance with other studies that observed the same finding at the end of laser application in spite of the use of different treatment protocol. ${ }^{(18,23-28)}$ In the present study, laser group showed insignificant decrease in pain intensity by the end of the study relative to the baseline indicating unsatisfactory long-term analgesic effect. This is in agreement with that of Ilbuldu et al. ${ }^{(18)}$ who found that laser therapy significantly decreased pain intensity, but this effect was not seen at the 6-month control. They concluded that laser therapy is perhaps effective in the short term, but the positive effects do not last. The present findings compare favorably to that of Fouda et al. ${ }^{(29)}$ In their study, the MTrP in the masseter muscle was exposed to low level laser in three sessions per week for 2 weeks using the diode laser with same properties as that used in the current study. They found insignificant decrease in pain intensity at the end of treatment and after 3 months. This could be attributed to the shorter treatment protocol used in their study.

With the exception of statistically significant decrease in mean PP scores, dry needling group showed insignificant decrease in pain intensity at the end of treatment and after 3 months in the current study. This is somewhat different from that of Ilbuldu et al. ${ }^{(18)}$ who found that dry needling had no effect on pain control. On the other hand, the present finding contradicts those of other studies. ${ }^{(30,31)}$ Ay et al ${ }^{(30)}$ found statistically significant improvements 
in pain VAS scores after 4 and 12 weeks of dry needling of trigger points in 40 patients compared to pre-treatment results and concluded that dry needling was effective in decrease of pain level in MPS. Gonzalez-Perez et al ${ }^{(31)}$ evaluated the usefulness of deep dry needling in the treatment of temporomandibular myofascial pain. They found the magnitude of pain reduction was statistically significant $(\mathrm{p}<0.01)$ in all controls performed. This contradiction could be attributed to the differences in the used needles type and treatment protocol.

There is a paucity of studies about the effect of LLLT and dry needling of MTrPs in masticatory muscles on mandibular function. There was no statistically significant change in mean MMO through all periods in both groups in the current study. This is in general agreement with that of Fouda et al. ${ }^{(29)}$ who found insignificant difference in MMO after LLLT of MTrP in the masseter muscle.

The results of the present study demonstrated that all evaluated parameters showed insignificant differences between laser and dry needling groups throughout the study intervals. However, laser group showed statistically significantly higher mean $\%$ decrease in pain scores at rest at the end of treatment than dry needling group. While at the end of the study dry needling group showed statistically significantly higher mean $\%$ decrease in pain scores on function than Laser group. Only one study performed by Ilbuldu et al. ${ }^{(18)}$ was found comparing low-level laser therapy and dry needling for the deactivation of MTPs. In contrast to the present finding, they observed a significant decrease in pain at rest, at activity, and increase in pain threshold in the laser group compared to dry needling group at post-treatment. When cervical range of motion was assessed, the range in extension was significantly increased in the laser group compared to dry needling. However, those differences among the groups were not observed at 6-month follow up.

\section{CONCLUSION}

Within the limitations of this study, it is possible to conclude that dry needling was equivalent to laser therapy for the deactivation of MTrPs. Nevertheless, laser therapy is perhaps effective in the short term, but the positive effects do not last. The insignificant pain reduction after 3 months in both groups suggests that the key to successful long-term outcomes of any treatment regime is addressing the precipitating and predisposing factors for each particular patient.

\section{REFERENCES}

1. Kamanli A, Kaya A, Ardicoglu O, Ozgocmen S, Zengin FO and Bayik Y: Comparison of lidocaine injection, botulinum toxin injection, and dry needling to trigger points in myofascial pain syndrome. Rheumatol Int. 2005;25:604-611.

2. Fricton JR, Kroening R, Haley D and Siegert R: Myofascial pain syndrome of the head and neck: a review of clinical characteristics of 164 patients.Oral Surg Oral Med Oral Pathol.1985;60:615-623.

3. Simons DG, Travell JG and Simons LS: Travell \& Simons' Myofascial pain and dysfunction: The trigger point manual vol. 1 and 2. Baltimore: Williams \& Wilkins.1999.

4. Truelove E, Sommers EE, LeResche L, Dworkin SF and Von Korff F: Clinical diagnostic criteria for TMD, new classification permits multiple diagnoses. J Am Dent Asso. 1992;123(4):47-54.

5. Dommerholt J: Myofascial Pain Syndrome in Craniomandibular Region. In: Padrós Serrat, E. (ed) Basis diagnostic, therapeutic and functional postural craniofacial. 2006;564-581.

6. Fricton $\mathrm{J}$, Kroening $\mathrm{R}$ and Hathaway $\mathrm{K}$ : $\mathrm{TMJ}$ and craniofacial pain: diagnosis and management. St. Louis: Ishiyaku EuroAmerica. 1988, $1^{\text {st }}$ edition.

7. McNeill C: Temporomandibular disorders: guidelines for classification, assessment, and management. Chicago: Quintessence Books. 1993, $1^{\text {st }}$ edition.

8. De Leeuw R: Orofacial pain guidelines for assessment, diagnosis and management. Quintessence Publishing. 2008, Vol 131, $4^{\text {th }}$ edition,1-59,129-204. 
9. Zohn DA and Mennell JM: Musculoskeletal pain: diagnosis and physical treatment, little, Brown, Boston. 1976;126-137.

10. Hou CR, Tsai LC, Cheng KF, Chung KC and Hong $\mathrm{CZ}$ : Immediate effects of various physical therapeutic modalities on cervical myofascial pain and trigger point sensitivity. Archives of Physical Medicine and Rehabilitation. 2002;83(10):1406 -1414.

11. Murphy GJ: Physical medicine modalities and trigger point injections in the management of temporomandibular disorders and assessing treatment outcome. Oral Surg. Oral Med. Oral Pathol. Oral Radiol. Endod. 1997;83,118-122.

12. Jaeger B and Reeves JL: Quantification of changes in myofascial trigger point sensitivity with the pressure algometer following passive stretch. Pain. 1986;27, 203210 .

13. Lewit K.: The needle effect in the relief of myofascial pain. Pain 1979;6(1): 83-90.

14. Baldry PE.: Superficial versus deep dry needling. Acupunct Med. 2002;20(2-3):78-81.

15. Tough EA, White AR, Cummings TM, Richards SH and Campbell JL:: Acupuncture and dry needling in the management of myofascial trigger point pain: A systematic review and meta-analysis of randomised controlled trials. European Journal of Pain 13. 2009; 3-10.

16. Smith H: Current Therapy in Pain: Expert Consult: Online and Print. Saunders, USA, Elsevier Health Sciences. $2008,1^{\text {st }}$ edition.

17. Kulekcioglu S, Sivrioglu K, Ozcan O and Parlak M: Effectiveness of low-level laser therapy in temporomandibular disorder. Scand. J. Rheumatol. 2003;32:114-118.

18. Ilbuldu E, Cakmak A, Disci R and Aydin R: Comparation of laser, dry needling, and placebo laser treatments in myofascial pain syndrome. Photomed Laser Surg. 2004;22(4):306-311.

19. Bjordal JM, Couppe C, Chow RT, Tuner J and Ljunggren AE: A systematic review of low level laser therapy with location-specific doses for pain from chronic joint disorders. Aust. J. Physiother. 2003;49:107-116.

20. Simunovic Z: Low level laser therapy with trigger points technique: A clinical study on 243 patients. J Clin Laser Med Surg. 1996;14(4):163-7.
21. Ziaeifar M, Arab AM, Karimi N and Nourbakhsh MR.: The effect of dry needling on pain, pressure pain threshold and disability in patients with a myofascial trigger point in the upper trapezius muscle. J Body w Mov Ther. 2014 Apr; 18(2):298-305.

22. Ozdemir F, Birtane M and Kokino S.: The clinical efficacy of low power laser therapy on pain and function in cervical osteoarthritis. Clin Rheumatol. 2001;20(3):181-184.

23. Hakguder A, Birtane M, Gurcan S, Kikino S and Turan FN: Efficacy of low level laser therapy in myofascial pain syndrome: an algometric and thermographic evaluation. Laser Surg Med. 2003;33(5): 339-343.

24. Ceccherelli F, Altafini L, Lo Castro G, Avila A, Ambrosio $\mathrm{F}$ and Giron GP: Diode laser in cervical myofascial pain: A double blind study versus placebo. Clin J Pain. 1989;5(4):301-304.

25. Azizi A, Sahebjamee M, Lawaf S, Jamalee F and Maroofi N: Effects of low-level laser in the treatment of myofascial pain_dysfunction syndrome. J Dental Research, Dental clinics, Dental prospects 2007; 1(2).

26. Uemoto L, Marco AC, Cresus VD, Oswaldo VV and Thays AA: Laser therapy and needling in myofascial trigger point deactivation. Journal of oral science. 2013;55(2):175-181.

27. Kiralp MZ, Ari H and Karabekir I.: Comparison of low intensity laser therapy and trigger point injection in the management of myofascial pain syndrome. Pain Clinic. 2006;18:63-6.

28. Venezian GC, da Silva MA, Mazzetto RG and Mazzetto MO.: Low level laser effects on pain to palpation and electromyographic activity in TMD patients: a doubleblind, randomized, placebo-controlled study. Cranio. 2010 Apr;28(2):84-91.

29. Fouda AA, Refai H, Mohammed NH: Low level laser therapy versus pulsed electromagnetic field for inactivation of myofascial trigger points. American Journal of Research Communication.2013;1(3): 68-78.

30. Ay S, Evcik D and Tur BS: Comparison of injection methods in myofascial pain syndrome: a randomized controlled trial. Clin Rheumatol. 2010;29:19-23.

31. Gonzalez-Perez LM, Infante-Cossio P, Granados-Nuñez M, Urresti-Lopez F J.: Treatment of temporomandibular myofascial pain with deep dry needling. Med Oral Patol Oral Cir Bucal.2012;17(5):781-5 